\title{
ESTUDO DA INTERAÇÃO DO HERBICIDA PICLORAM COM AMOSTRAS DE SOLO DO SEMIÁRIDO BAIANO
}

\author{
$\underline{\text { Alessandra de Jesus Silva }}{ }^{1}$; Luciana Bagdeve de Oliveira dos Santos ${ }^{2}$; Lilian \\ Graicy Lima Morais Lima ${ }^{3}$ e Maria do Socorro Costa São Mateus ${ }^{4}$ \\ 1. Bolsista PIBIC/Fapesb, Graduanda em Licenciatura em Química, Universidade Estadual de Feira de Santana, e- \\ mail: allessandrafsa@hotmail.com \\ 2. Orientador, Departamento de Exatas, Universidade Estadual de Feira de Santana, e-mail: lbagdeve@uefs.br \\ 3. Mestre em Ciências Ambientais e Doutoranda em Geologia, Universidade Estadual de Feira de Santana, e-mail: \\ graicylima@gmail.com \\ 4. Participante do Mestrado PPGECEA/Uefs, Departamento de Tecnologia, Universidade Estadual de Feira de \\ Santana, e-mail: somateus@gmail.com
}

PALAVRAS-CHAVE: Adsorção; Picloram; Solo.

\section{INTRODUÇÃOO}

O crescimento da produção de alimentos não teria sido possível sem o uso dos defensivos agrícolas como os pesticidas. Esses compostos orgânicos, também conhecidos como agrotóxicos, tiveram avanço considerável desde a sua descoberta durante a segunda guerra mundial. As classes mais conhecidas desses compostos sintéticos utilizados como pesticidas são os organoclorados, organofosforados, os nitrocompostos, os piridínicos, as triazinas, carbamatos e piretróides (Barbosa, 2004; Garrido et al, 2004). Tais compostos podem interagir com argilas e a matéria orgânica presentes no solo, reduzindo sua potencialidade no combate às pragas, uma vez que a fração disponível do pesticida será menor. Tal desvantagem pode ocasionar, mesmo quando empregados de modo correto, danos à saúde pública e/ou ambiental. O pesticida picloram (ácido 4-amino-3,5,6-tricloropicolínico), da classe dos organoclorados é classificado como herbicida, por ser utilizado para auxiliar no controle de plantas daninhas que afetam o plantio. É considerado um herbicida ácido (Spadotto et al, 2003), cujo $\mathrm{pK}=3,4$. Em $\mathrm{pHs}$ mais baixos que 3,4, o pesticida se encontrará predominantemente na forma molecular e acima desse $\mathrm{pH}$, cresce a fração iônica do pesticida.

O solo é um material granular, constituído na sua maior parte por matéria sólida (orgânica e mineral), além das fases líquida e gasosa. Um perfil de solo é o conjunto de todos os horizontes ou camadas que constituem o perfil adicionados de material mineral e resíduos orgânicos, e estes horizontes subdividem-se em superficiais $(\mathrm{O}$ ou $\mathrm{H} \mathrm{e} \mathrm{A})$ e subsuperficiais (E, B, C, F e R) com características diferenciadas entre si, podendo ainda existir horizontes intermediários ou transicionais (AB, EB, BC, etc). Uma vez aplicado no combate às pragas, o picloram pode, a depender de condições do solo como: quantidade de matéria orgânica e argilominerais, $\mathrm{pH}$, força iônica, etc, interagir com o solo ou ser lixiviado através dele e causar a contaminação de fontes de água, superficiais ou subterrâneas, utilizadas para abastecimento humano e manutenção de atividades econômicas. Portanto, o estudo da interação do picloram em diferentes horizontes de um perfil de solo propicia o entendimento das interações e fenômenos de deslocamento do herbicida no mesmo, através de isotermas de adsorção sob diferentes condições de $\mathrm{pH}$ e força iônica.

\section{METODOLOGIA}

\section{Coleta e preparo das amostras de solo}

As amostras de solo foram coletadas no campus da Universidade Estadual de Feira de Santana (UEFS) em Feira de Santana, Bahia, Brasil, sem histórico de aplicação de pesticidas, conforme o conforme o Manual de descrição e coleta de solo no campo 
(Lemos, 1996). Foram coletadas amostras de dois horizontes de um perfil de solo de profundidade de $100 \mathrm{~cm}$, horizonte A $(0-40 \mathrm{~cm})$ e horizonte $A B(40-100 \mathrm{~cm})$. As amostras foram preparadas de acordo com a Norma Brasileira - NBR 6457/86.

Caracterização e classificação das amostras de solo

A caracterização das amostras de solo quanto à cor foi realizada utilizando-se a caderneta de cores Munsell Soil-Color Charts. A análise granulométrica por peneiramento e sedimentação, para a obtenção da textura das amostras, foi realizada conforme as especificações da Norma Brasileira - NBR 7181/84. A classificação das amostras de solo caracterizadas adotada foi a da Associação Brasileira de Normas Técnicas (ABNT) para solos, prevista na Norma Brasileira - NBR 6502/95. A caracterização mineralógica das amostras foi feita empregando-se a espectrorradiometria utilizando o Espectrorradiômetro portátil modelo ASD-FieldSpec Full Resolution 3. Os dados obtidos foram visualizados no software ENVI® e comparadas com a biblioteca espectral de referência.

\section{Estudos de adsorção do pesticida no solo em diferentes meios e análises cromatográficas}

Os estudos de adsorção envolvendo o picloram em solo foram realizados em $\mathrm{CaCl}_{2}$ 0,01 mol L ${\mathrm{e} \mathrm{HNO}_{3} \text { 0,01 mol L }}^{-1}$, em sistema de batelada, de acordo com as recomendações do Institute for Reference Materials and Measurements e da Organization for Economic Cooperation and Development (OECD,2000). A proporção entre massa de solo e volume de solução foi de $1 \mathrm{~g}$ : $5 \mathrm{~mL}$. Os resíduos de picloram foram determinados empregando Cromatografia Líquida de Alta Eficiência (HPLC), em modo de eluição isocrático. Para as amostras obtidas em $\mathrm{CaCl}_{2} 0,01 \mathrm{~mol} \mathrm{~L}^{-1}$ a fase móvel era constituída por $50 \%$ (v/v) em solução de ácido fosfórico $0,1 \%(\mathrm{~m} / \mathrm{v})$ e acetonitrila. Para as amostras obtidas em $\mathrm{HNO}_{3} \quad 0,01 \mathrm{~mol} \mathrm{~L}^{-1}$ a fase móvel era constituída por $60 \%(\mathrm{v} / \mathrm{v})$ em solução de ácido fosfórico $0,1 \%(\mathrm{~m} / \mathrm{v})$ e acetonitrila. A detecção foi realizada empregando detector UV que monitorou absorbância em $220 \mathrm{~nm}$. As equações de Langmuir ou Freundlich foram utilizadas para interpretar os dados experimentais obtidos.

\section{RESULTADOS E DISCUSSÃO}

O preparo e caracterização das amostras de solo foram feitas nos laboratórios de pedologia, geoquímica e catálise ambiental, geotecnia e espectrorradiometria da UEFS. A Tabela 1 mostra os resultados obtidos.

Tabela 1. Características das amostras de solo estudadas.

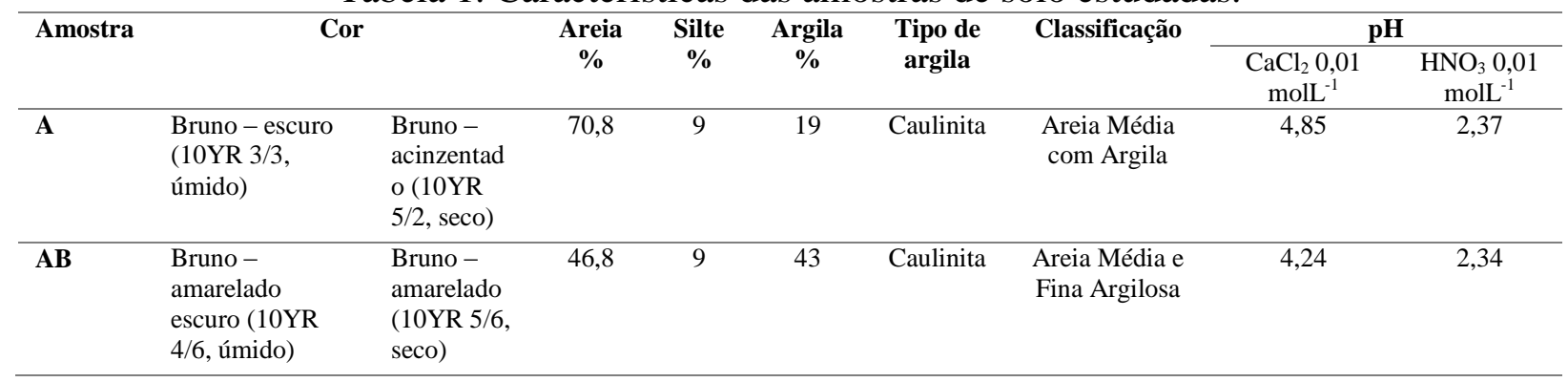

A o resultado da análise espectral para as amostras dos horizontes $\mathrm{A}$ e $\mathrm{AB}$ do perfil de solo coletado indicou a presença do argilomineral caulinita nos horizontes citados. Observou-se a presença das bandas de absorção dupla em aproximadamente 1.4 micrômetros e 2.2 micrômetros. Essas feições são típicas da presença de caulinita na amostra, bastante ocorrente em solos tropicais. 


\section{Análise de picloram em extrato de solo e Isotermas de adsorção}

Foram estudados os tempos de equilíbrio para os sistemas pesticida- perfis de solo em meio de $\mathrm{CaCl}_{2}$ 0,01 mol L $\mathrm{L}^{-1}{\mathrm{e} \mathrm{HNO}_{3}}_{0,01} \mathrm{~mol} \mathrm{~L} \mathrm{~L}^{-1}$. Os sistemas estudados atingiram o equilíbrio em diferentes tempos, por questões operacionais, todos os experimentos de adsorção foram realizados utilizando $24 \mathrm{~h}$ como tempo de equilíbrio, uma vez que neste tempo observamos que os valores das concentrações do pesticida livre, no equilíbrio, não sofreram alterações significativas.

A determinação de picloram em extrato de solo na presença de $\mathrm{CaCl}_{2} 0,01 \mathrm{molL} \mathrm{L}^{-}$ ${ }^{1}$ ou $\mathrm{HNO}_{3}$ 0,01 molL ${ }^{-1}$ foi realizada após adição de quantidade conhecida do herbicida, que variou entre 4,1 e $207,1 \mu \mathrm{mol} \mathrm{\textrm {L } ^ { - 1 }}$. Os resultados obtidos para os sistemas em $\mathrm{CaCl}_{2}$ $0,01 \mathrm{~mol} \mathrm{~L}^{-1}$ e em $\mathrm{HNO}_{3} 0,01 \mathrm{~mol} \mathrm{~L}^{-1}$ são apresentados na Tabela 2. Tais resultados consistem nos valores obtidos da média de duas amostras com seus respectivos desvios.

Tabela 2. Valores de concentração livre de picloram em extrato de solo na presença de

$\mathrm{CaCl}_{2}$ 0,01 $\mathrm{mol} \mathrm{L}^{-1}$ e $\mathrm{HNO}_{3}$ 0,01 mol L-1, obtidos por HPLC, após 24 horas de equilíbrio do pesticida com amostras dos horizontes $\mathrm{A}$ e $\mathrm{AB}$ do solo estudado. Estudo

\begin{tabular}{|c|c|c|c|c|c|c|c|c|}
\hline $\begin{array}{c}\mathrm{C}_{\text {inic. Pic. }} \\
\left(\mu \mathrm{mol} \mathrm{L} \mathrm{L}^{-1}\right)\end{array}$ & $\begin{array}{c}\text { (HPLC) } \\
\text { C }_{\text {pic. livre }} \\
(\mu \mathrm{mol} \mathrm{L} \\
\end{array}$ & $\begin{array}{c}\text { Desvio } \\
\left(\mu \mathrm{mol} \mathrm{L}{ }^{-1}\right)\end{array}$ & $\begin{array}{c}\text { (HPLC) } \\
\mathrm{C}_{\text {pic. livre }} \\
\left(\mu \mathrm{mol} \mathrm{L}^{-1}\right)\end{array}$ & $\begin{array}{c}\text { Desvio } \\
\left(\mu \mathrm{mol} \mathrm{L}{ }^{-1}\right)\end{array}$ & $\begin{array}{c}\text { (HPLC) } \\
\mathrm{C}_{\text {pic. livre }} \\
\left.(\mu \mathrm{mol} \mathrm{L})^{-1}\right)\end{array}$ & $\begin{array}{c}\text { Desvio } \\
\left.(\mu \mathrm{mol} \mathrm{L})^{-1}\right)\end{array}$ & $\begin{array}{c}\text { (HPLC) } \\
\text { C }_{\text {pic. livre }} \\
\left.(\mu \mathrm{mol} \mathrm{L})^{-1}\right)\end{array}$ & $\begin{array}{c}\text { Desvio } \\
\left.(\mu \mathrm{mol} \mathrm{L})^{-1}\right)\end{array}$ \\
\hline & \multicolumn{4}{|c|}{$\mathrm{CaCl}_{2} 0,01 \mathrm{~mol} \mathrm{~L}^{-1}$} & \multicolumn{4}{|c|}{$\mathrm{HNO}_{3} 0,01 \mathrm{~mol} \mathrm{~L}^{-1}$} \\
\hline & \multicolumn{2}{|l|}{ Horizonte A } & \multicolumn{2}{|c|}{ Horizonte AB } & Horizonte A & \multicolumn{3}{|c|}{ Horizonte AB } \\
\hline 4,1 & 3,2 & 0,1 & 3,8 & 0,1 & 2,94 & 0,03 & 2,8 & 0,3 \\
\hline 8,3 & 7,6 & 0,1 & 8,1 & 0,2 & 6,3 & 0,4 & 6,0 & 0,3 \\
\hline 16,6 & 16,7 & 0,2 & 14 & 3 & 13 & 1 & 13 & 1 \\
\hline 24,8 & 24 & 1 & 24,01 & 0,01 & 20 & 2 & 20,3 & 0,3 \\
\hline 33,1 & 32 & 2 & 32 & 1 & 27 & 1 & 27 & 2 \\
\hline 49,7 & 48 & 1 & 48,4 & 0,2 & 39,7 & 0,4 & 40 & 1 \\
\hline 103,5 & 102 & 2 & 98 & 3 & 83 & 1 & 84 & 2 \\
\hline 207,1 & 201 & 2 & 197 & 4 & 181 & 4 & 170 & 4 \\
\hline
\end{tabular}

Os resultados obtidos, conforme mostrados na Tabela 2, foram utilizados posteriormente para obtenção das isotermas de adsorção de picloram em solo. Os parâmetros de Freundilich e $\mathrm{R}^{2}$ são apresentados na Tabela 3.

Tabela 3. Parâmetros de Freundlich $K_{f}\left(\mu \mathrm{mol}{ }^{1-1 / n} \mathrm{~L}^{1 / n} \mathrm{~g}^{-1}\right)$ e $1 / \mathrm{n}$ para adsorção de picloram em amostras A e AB do perfil de solo do semiárido baiano. Faixa de concentração inicial estudada: 4,1 a $207,1 \mu \mathrm{mol} \mathrm{L}{ }^{-1}$.

\begin{tabular}{lcccccccc}
\hline Amostra & \multicolumn{3}{c}{ Adsorção em $\mathbf{C a C l}_{\mathbf{2}} \mathbf{0 , 0 1} \mathbf{~ m o l L}^{-\mathbf{1}}$} & \multicolumn{4}{c}{ Adsorção em $\mathbf{H N O}_{\mathbf{3}} \mathbf{0 , 0 1} \mathbf{~ m o l L ~}^{-\mathbf{1}}$} \\
\hline & $\mathbf{K}_{\mathbf{f}}$ & $\mathbf{1 / n}$ & $\mathbf{n}$ & $\mathbf{R}^{\mathbf{2}}$ & $\mathbf{K}_{\mathbf{f}}$ & $\mathbf{1 / n}$ & $\mathbf{n}$ & $\mathbf{R}^{\mathbf{2}}$ \\
\hline $\mathbf{A}$ & $(1,0 \pm 0,8) \times 10^{-3}$ & $1,3 \pm 0,1$ & $0,8 \pm 0,1$ & 0,696 & $(2,4 \pm 0,5) 10^{-3}$ & $0,80 \pm 0,04$ & $1,3 \pm 0,1$ & 0,981 \\
\hline $\mathbf{A B}$ & $(6 \pm 3) \times 10^{-5}$ & $0,85 \pm 0,06$ & $1,2 \pm 0,1$ & 0,960 & $(3,3 \pm 0,4) 10^{-3}$ & $0,76 \pm 0,03$ & $1,3 \pm 0,1$ & 0,989 \\
\hline
\end{tabular}

Observou-se valores de $\mathrm{n}$ maiores para os experimentos realizados em $\mathrm{HNO}_{3}$ ( $\mathrm{pH}=2,37$ horizonte $\mathrm{A}$ e $\mathrm{pH}=2,34$ horizonte $\mathrm{AB}$ ) quando comparados aos experimentos realizados em $\mathrm{CaCl}_{2}$, que podem ser atribuídos ao picloram encontrar-se majoritariamente na forma molecular em $\mathrm{pHs}$ mais baixos. $\mathrm{O}$ parâmetro $\mathrm{n}$ está relacionado à intensidade de adsorção entre adsorvente e adsorvato. Uma adsorção favorável tem valores de $\mathrm{n}$ entre 1 e 10. Assim, moléculas de picloram possuem maior afinidade por partículas carregadas da argila e substâncias húmicas que o pesticida na 
forma aniônica. Podemos também atribuir as maiores capacidades de adsorção $\left(\mathrm{K}_{\mathrm{f}}\right.$ Tabela 3) do picloram pelo adsorvato, neste meio, pelo mesmo motivo. Para os estudos conduzidos em $\mathrm{HNO}_{3}$, no horizonte A é observada adsorção um pouco menor que em relação horizonte $\mathrm{AB}$, isto porque, de acordo com a sua textura, esta camada superficial do perfil de solo é constituída por mais areia e menor fração do argilomineral caulinita (19\%), enquanto no horizonte AB são encontrados $43 \%$ do argilomineral. Solos mais argilosos geralmente possuem maior capacidade de retenção do que os solos mais arenosos.

\section{CONCLUSÃO}

Os parâmetros de adsorção ( $\left.\mathrm{K}_{\mathrm{f}} \mathrm{e} n\right)$ encontrados para os estudos conduzidos entre o herbicida picloram em horizontes do perfil de solo do semiárido baiano, $\mathrm{A}$ e $\mathrm{AB}$, indicaram que o comportamento do pesticida irá diferir a depender do $\mathrm{pH}$ do meio e características do solo, como teor de argilas.

Os valores de $\mathrm{n}$ e $\mathrm{K}_{\mathrm{f}}$ determinados através das isotermas de Freundlich, indicaram adsorção favorável e melhor capacidade de retenção do picloram nos perfis de solos $\mathrm{A}$ e $\mathrm{AB}$ para $\mathrm{pH}$ menor do que três (experimentos realizados em $\mathrm{HNO}_{3}$ ), esse fato pode ser atribuído à forma química em que se encontra o pesticida, predominantemente como moléculas. Observa-se também maior capacidade de adsorção $\left(\mathrm{K}_{\mathrm{f}}\right)$ para o solo com mais alto teor de argila, horizonte AB (43\%). Isso implica que ao ser aplicado em solos cujos pHs estejam acima do $\mathrm{pKa}$ do picloram, uma menor fração do pesticida seria retida e em consequência teríamos maior mobilidade do pesticida no meio podendo, por lixiviação, contaminar águas naturais subterrâneas como rios, lagos e lençóis freáticos próximos à aplicação do herbicida.

\section{REFERÊNCIAS}

ABNT - Associação Brasileira de Normas Técnicas. 1984. Solo - Análise granulométrica - NBR 7181.

ABNT - Associação Brasileira de Normas Técnicas. 1984. Determinação da massa específica - NBR 6508.

ABNT - Associação Brasileira de Normas Técnicas. 1986. Solo - Preparação para ensaios de compactação e ensaios de caracterização - NBR 6457.

ABNT - Associação Brasileira de Normas Técnicas. 1995. Rochas e solos - NBR 6502. BARBOSA, L.C.A. 2004. Os pesticidas, o homem e o meio ambiente. Univ. Federal de Viçosa, p.1-207.

CAPUTO, H. P. 1988. Mecânica dos Solos e suas Aplicações. Rio de Janeiro, Livros Técnicos e Científicos Editora, p. 14-60, 183-185.

GARRIDO, E.M.; DELERUE, M. C.; LIMA, J.L.F.C.; BRETT, A.M.O., Electrochemical methods in pesticides control. 2004. Analytical Letters. 37(9): 17551791.

MUNSELL COLOR. 1975. Munsell soil color charts, Baltimore.

SANTOS, L. B.O dos; ABATE, G.; MASINI, J. C. 2005. Application of sequential injection-square wave voltammetry (SI-SWV) to study the adsorption of atrazine onto a tropical soil sample. Talanta. 68: 165-170.

SPADOTTO, C.A.; HORNSBY, A.G. 2003. Soil sorption of pesticides: modeling pH effects. J. Environ. Qual. 32: 949.

LEMOS, R. C. 1996. Manual de descrição e coleta de solo no campo. Campinas, Sociedade Brasileira de Ciência do Solo, 84p.

OECD guide line for the testing of chemicals, Adopted : 21 de Janeiro 2000.

ITT Visual Information Solutions. ENVI Version 4.7 (C) 2009. 